\title{
On a variant of Čebyšev's inequality of the Mercer type
}

\section{Anita Matković ${ }^{*}$ (1) and Josip Pečarić ${ }^{2}$ (1)}

Dedicated to Professor Shoshana Abramovich on the occasion of her 80th birthday.

\section{"Correspondence:}

anita.matkovic@fesb.hr

${ }^{1}$ Faculty of Electrical Engineering,

Mechanical Engineering and Naval

Architecture, University of Split,

Rudjera Boškovića 32, 21000 Split,

Croatia

Full list of author information is

available at the end of the article

\begin{abstract}
We consider the discrete Jensen-Mercer inequality and Čebyšev's inequality of the Mercer type. We establish bounds for Čebyšev's functional of the Mercer type and bounds for the Jensen-Mercer functional in terms of the discrete Ostrowski inequality. Consequentially, we obtain new refinements of the considered inequalities.
\end{abstract}

MSC: 26D15

Keywords: Jensen-Mercer inequality; Čebyšev's inequality of the Mercer type

\section{Introduction}

Let $n \geq 2$ and let $\boldsymbol{w}=\left(w_{1}, \ldots, w_{n}\right)$ be a real $n$-tuple such that

$$
0 \leq W_{k}=\sum_{i=1}^{k} w_{i} \leq W_{n}, \quad k=1, \ldots, n, W_{n}>0
$$

In [5] the following Čebyšev's inequality of the Mercer type:

$$
\left(a+b-\frac{1}{W_{n}} \sum_{i=1}^{n} w_{i} x_{i}\right)\left(c+d-\frac{1}{W_{n}} \sum_{i=1}^{n} w_{i} y_{i}\right) \leq a c+b d-\frac{1}{W_{n}} \sum_{i=1}^{n} w_{i} x_{i} y_{i},
$$

was proved for any real $n$-tuples $\boldsymbol{x}=\left(x_{1}, \ldots, x_{n}\right)$ and $\boldsymbol{y}=\left(y_{1}, \ldots, y_{n}\right)$ monotone in the same direction and real numbers $a, b, c, d$ such that

$$
a \leq \min _{1 \leq i \leq n} x_{i}, \quad b \geq \max _{1 \leq i \leq n} x_{i}, \quad c \leq \min _{1 \leq i \leq n} y_{i}, \quad d \geq \max _{1 \leq i \leq n} y_{i} .
$$

If $\boldsymbol{x}$ and $\boldsymbol{y}$ are monotonic in the opposite directions, inequality (2) is reversed.

Here, to be more precise, we cite that result with the slightly different notation.

In the same paper, the authors considered Čebyšev's functional (or Čebyšev's difference) of the Mercer type defined as the difference of the right- and left-hand sides of inequality (2). They established bounds in terms of the discrete Ostrowski inequality. Here we give

(c) The Author(s) 2020. This article is licensed under a Creative Commons Attribution 4.0 International License, which permits use, sharing, adaptation, distribution and reproduction in any medium or format, as long as you give appropriate credit to the original author(s) and the source, provide a link to the Creative Commons licence, and indicate if changes were made. The images or other third party material in this article are included in the article's Creative Commons licence, unless indicated otherwise in a credit line to the material. If material is not included in the article's Creative Commons licence and your intended use is not permitted by statutory regulation or exceeds the permitted use, you will need to obtain permission directly from the copyright holder. To view a copy of this licence, visit http://creativecommons.org/licenses/by/4.0/. 
more accurate bounds, which also provide refinements of inequality (2). In addition, using these results, we establish Ostrowski-like bounds for the Jensen-Mercer functional and, consequentially, a refinement of the Jensen-Mercer inequality.

\section{Bounds for the Čebyšev's functional of the Mercer type}

Let $m \geq 2$ and let $\boldsymbol{p}=\left(p_{1}, \ldots, p_{m}\right)$ be a real $m$-tuple such that

$$
0 \leq P_{k}=\sum_{i=1}^{k} p_{i} \leq P_{m}, \quad k=1, \ldots, m, P_{m}>0 .
$$

Then $\bar{P}_{k}=\sum_{i=k}^{m} p_{i} \geq 0, k=1, \ldots, m$. Furthermore, from the summation by parts (sometimes called the Abel transformation) it follows that the identity

$$
\begin{aligned}
& \sum_{i=1}^{m} p_{i} \sum_{i=1}^{m} p_{i} \xi_{i} \zeta_{i}-\sum_{i=1}^{m} p_{i} \xi_{i} \sum_{i=1}^{m} p_{i} \zeta_{i} \\
& =\sum_{i=1}^{m-1}\left(\sum_{j=1}^{i-1} \bar{P}_{i+1} P_{j} \Delta \xi_{i} \Delta \zeta_{j}+\sum_{j=i}^{m-1} P_{i} \bar{P}_{j+1} \Delta \xi_{i} \Delta \zeta_{j}\right)
\end{aligned}
$$

holds for any two real $m$-tuples $\xi=\left(\xi_{1}, \ldots, \xi_{m}\right)$ and $\zeta=\left(\zeta_{1}, \ldots, \zeta_{m}\right)$, where $\Delta \xi_{i}=\xi_{i+1}-\xi_{i}$, $\Delta \zeta_{i}=\zeta_{i+1}-\zeta_{i}, i=1, \ldots, m-1$ (see $[7,8]$ ).

Here, and in the rest of the paper, we assume $\sum_{j=k}^{l} x_{j}=0$ when $k>l$.

Lemma 1 Let $n \geq 2$ and let $\boldsymbol{w}$ be a real $n$-tuple such that (1) is fulfilled. Then for any real $n$-tuples $\boldsymbol{x}, \boldsymbol{y}$ and real numbers $a, b, c, d$ satisfying (3), the identity

$$
\begin{aligned}
& a c+ b d-\frac{1}{W_{n}} \sum_{i=1}^{n} w_{i} x_{i} y_{i}-\left(a+b-\frac{1}{W_{n}} \sum_{i=1}^{n} w_{i} x_{i}\right)\left(c+d-\frac{1}{W_{n}} \sum_{i=1}^{n} w_{i} y_{i}\right) \\
&=\left(x_{1}-a\right)\left(d-y_{n}\right)+\left(b-x_{n}\right)\left(y_{1}-c\right) \\
&+\frac{1}{W_{n}}\left[\sum_{i=1}^{n-1} W_{i}\left(x_{1}-a\right) \Delta y_{i}+\sum_{i=1}^{n-1} \bar{W}_{i+1}\left(b-x_{n}\right) \Delta y_{i}\right. \\
&\left.+\sum_{i=1}^{n-1} W_{i} \Delta x_{i}\left(y_{1}-c\right)+\sum_{i=1}^{n-1} \bar{W}_{i+1} \Delta x_{i}\left(d-y_{n}\right)\right] \\
&+\frac{1}{W_{n}^{2}} \sum_{i=1}^{n-1}\left(\sum_{j=1}^{i-1} W_{i} \bar{W}_{j+1} \Delta x_{i} \Delta y_{j}+\sum_{j=i}^{n-1} \bar{W}_{i+1} W_{j} \Delta x_{i} \Delta y_{j}\right)
\end{aligned}
$$

holds, where $\Delta x_{i}=x_{i+1}-x_{i}, \Delta y_{i}=y_{i+1}-y_{i}, i=1, \ldots, n-1$.

Proof For $m=n+2$, we define $m$-tuples $\boldsymbol{p}, \boldsymbol{\xi}$, and $\zeta$ as

$$
\begin{array}{llll}
p_{1}=1, & p_{2}=-\frac{w_{1}}{W_{n}}, \quad p_{3}=-\frac{w_{2}}{W_{n}}, & \ldots, \quad p_{m-1}=-\frac{w_{n}}{W_{n}}, \quad p_{m}=1, \\
\xi_{1}=a, & \xi_{2}=x_{1}, \quad \xi_{3}=x_{2}, \quad \ldots, \quad \xi_{m-1}=x_{n}, \quad \xi_{m}=b, & \\
\zeta_{1}=c, & \zeta_{2}=y_{1}, \quad \zeta_{3}=y_{2}, \quad \ldots, & \zeta_{m-1}=y_{n}, \quad \zeta_{m}=d .
\end{array}
$$


Since $\boldsymbol{w}$ satisfies (1) it follows that

$$
0 \leq P_{k}=\sum_{i=1}^{k} p_{i} \leq P_{m}, \quad k=1,2, \ldots, m, P_{m}=1>0
$$

Hence, we can apply identity (5). Its left-hand side is

$$
\begin{aligned}
& \sum_{i=1}^{m} p_{i} \sum_{i=1}^{m} p_{i} \xi_{i} \zeta_{i}-\sum_{i=1}^{m} p_{i} \xi_{i} \sum_{i=1}^{m} p_{i} \zeta_{i} \\
& \quad=a c+b d-\frac{1}{W_{n}} \sum_{i=1}^{n} w_{i} x_{i} y_{i}-\left(a+b-\frac{1}{W_{n}} \sum_{i=1}^{n} w_{i} x_{i}\right)\left(c+d-\frac{1}{W_{n}} \sum_{i=1}^{n} w_{i} y_{i}\right)
\end{aligned}
$$

It can be easily seen that

$$
\begin{array}{ll}
P_{1}=1, & P_{m}=1, \quad P_{m-1}=0, \quad P_{i}=\frac{\bar{W}_{i}}{W_{n}}, \quad \text { for } i=2, \ldots, m-2, \\
\bar{P}_{1}=1, & \bar{P}_{m}=1, \quad \bar{P}_{2}=0, \quad \bar{P}_{i}=\frac{W_{i-2}}{W_{n}}, \quad \text { for } i=3, \ldots, m-1,
\end{array}
$$

hence, on the right-hand side of (5) we have

$$
\begin{aligned}
& \sum_{i=1}^{m-1}\left(\sum_{j=1}^{i-1} \bar{P}_{i+1} P_{j} \Delta \xi_{i} \Delta \zeta_{j}+\sum_{j=i}^{m-1} P_{i} \bar{P}_{j+1} \Delta \xi_{i} \Delta \zeta_{j}\right) \\
& =\sum_{i=1}^{m-1}\left(\bar{P}_{i+1} P_{1} \Delta \xi_{i} \Delta \zeta_{1}+\sum_{j=2}^{i-1} \bar{P}_{i+1} P_{j} \Delta \xi_{i} \Delta \zeta_{j}\right. \\
& \left.\quad+\sum_{j=i}^{m-2} P_{i} \bar{P}_{j+1} \Delta \xi_{i} \Delta \zeta_{j}+P_{i} \bar{P}_{m} \Delta \xi_{i} \Delta \zeta_{m-1}\right) \\
& =\sum_{i=1}^{m-1}\left(\bar{P}_{i+1} \Delta \xi_{i}\left(y_{1}-c\right)+\frac{1}{W_{n}} \sum_{j=2}^{i-1} \bar{P}_{i+1} \bar{W}_{j} \Delta \xi_{i} \Delta y_{j-1}\right. \\
& \left.\quad+\frac{1}{W_{n}} \sum_{j=i}^{m-2} P_{i} W_{j-1} \Delta \xi_{i} \Delta y_{j-1}+P_{i} \Delta \xi_{i}\left(d-y_{n}\right)\right)
\end{aligned}
$$

Calculating separately summands for $i=1$ and $i=m-1$, we obtain

$$
\begin{gathered}
\sum_{i=1}^{m-1}\left(\bar{P}_{i+1} \Delta \xi_{i}\left(y_{1}-c\right)+\frac{1}{W_{n}} \sum_{j=2}^{i-1} \bar{P}_{i+1} \bar{W}_{j} \Delta \xi_{i} \Delta y_{j-1}\right. \\
\left.+\frac{1}{W_{n}} \sum_{j=i}^{m-2} P_{i} W_{j-1} \Delta \xi_{i} \Delta y_{j-1}+P_{i} \Delta \xi_{i}\left(d-y_{n}\right)\right) \\
=\frac{1}{W_{n}} \sum_{j=2}^{m-2} W_{j-1}\left(x_{1}-a\right) \Delta y_{j-1}+\left(x_{1}-a\right)\left(d-y_{n}\right)
\end{gathered}
$$




$$
\begin{aligned}
& +\frac{1}{W_{n}} \sum_{i=2}^{m-2} W_{i-1} \Delta x_{i-1}\left(y_{1}-c\right)+\frac{1}{W_{n}^{2}} \sum_{i=2}^{m-2} \sum_{j=2}^{i-1} W_{i-1} \bar{W}_{j} \Delta x_{i-1} \Delta y_{j-1} \\
& +\frac{1}{W_{n}^{2}} \sum_{i=2}^{m-2} \sum_{j=i}^{m-2} \bar{W}_{i} W_{j-1} \Delta x_{i-1} \Delta y_{j-1}+\frac{1}{W_{n}} \sum_{i=2}^{m-2} \bar{W}_{i} \Delta x_{i-1}\left(d-y_{n}\right) \\
& +\left(b-x_{n}\right)\left(y_{1}-c\right)+\frac{1}{W_{n}} \sum_{j=2}^{m-2} \bar{W}_{j}\left(b-x_{n}\right) \Delta y_{j-1} .
\end{aligned}
$$

Therefore,

$$
\begin{aligned}
& \sum_{i=1}^{m-1}\left(\sum_{j=1}^{i-1} \bar{P}_{i+1} P_{j} \Delta \xi_{i} \Delta \zeta_{j}+\sum_{j=i}^{m-1} P_{i} \bar{P}_{j+1} \Delta \xi_{i} \Delta \zeta_{j}\right) \\
& =\left(x_{1}-a\right)\left(d-y_{n}\right)+\left(b-x_{n}\right)\left(y_{1}-c\right) \\
& \quad+\frac{1}{W_{n}}\left[\sum_{j=2}^{n} W_{j-1}\left(x_{1}-a\right) \Delta y_{j-1}+\sum_{j=2}^{n} \bar{W}_{j}\left(b-x_{n}\right) \Delta y_{j-1}\right. \\
& \left.\quad+\sum_{i=2}^{n} W_{i-1} \Delta x_{i-1}\left(y_{1}-c\right)+\sum_{i=2}^{n} \bar{W}_{i} \Delta x_{i-1}\left(d-y_{n}\right)\right] \\
& \quad+\frac{1}{W_{n}^{2}} \sum_{i=2}^{n}\left(\sum_{j=2}^{i-1} W_{i-1} \bar{W}_{j} \Delta x_{i-1} \Delta y_{j-1}+\sum_{j=i}^{n} \bar{W}_{i} W_{j-1} \Delta x_{i-1} \Delta y_{j-1}\right)
\end{aligned}
$$

which is equal to the right-hand side of (6).

Using identity (6) and imposing stricter conditions than (3), we obtain refinements of inequality (2) which are more accurate than those previously established in [5].

Theorem 1 Let $n \geq 2$ and let $\boldsymbol{w}$ be a real $n$-tuple such that (1) is fulfilled. Let $\boldsymbol{x}, \boldsymbol{y}$ be real $n$-tuples monotonic in the same direction. Suppose that real numbers $a, b, c, d$ and nonnegative real numbers $r$, s satisfy

$$
\begin{array}{lll}
\min _{1 \leq i \leq n} x_{i}-a \geq r, & b-\max _{1 \leq i \leq n} x_{i} \geq r, & \left|\Delta x_{i}\right| \geq r, \quad i=1, \ldots, n-1, \\
\min _{1 \leq i \leq n} y_{i}-c \geq s, & d-\max _{1 \leq i \leq n} y_{i} \geq s, & \left|\Delta y_{i}\right| \geq s, \quad i=1, \ldots, n-1 .
\end{array}
$$

Then

$$
\begin{aligned}
& a c+b d-\frac{1}{W_{n}} \sum_{i=1}^{n} w_{i} x_{i} y_{i}-\left(a+b-\frac{1}{W_{n}} \sum_{i=1}^{n} w_{i} x_{i}\right)\left(c+d-\frac{1}{W_{n}} \sum_{i=1}^{n} w_{i} y_{i}\right) \\
& \geq r s\left(2 n+\frac{1}{W_{n}^{2}} \sum_{i=1}^{n-1}\left(\sum_{j=1}^{i-1} W_{i} \bar{W}_{j+1}+\sum_{j=i}^{n-1} \bar{W}_{i+1} W_{j}\right)\right) \geq 0 .
\end{aligned}
$$

If $\mathbf{x}$ and $\mathbf{y}$ are monotonic in the opposite directions, then the inequalities in (10) are reversed and the term rs appears with the negative sign. 
Proof Under the given assumptions, using identity (6), we obtain

$$
\begin{aligned}
& a c+b d-\frac{1}{W_{n}} \sum_{i=1}^{n} w_{i} x_{i} y_{i}-\left(a+b-\frac{1}{W_{n}} \sum_{i=1}^{n} w_{i} x_{i}\right)\left(c+d-\frac{1}{W_{n}} \sum_{i=1}^{n} w_{i} y_{i}\right) \\
& \geq 2 r s+\frac{2 r s}{W_{n}}\left(\sum_{i=1}^{n-1} W_{i}+\sum_{i=1}^{n-1} \bar{W}_{i+1}\right)+\frac{r s}{W_{n}^{2}} \sum_{i=1}^{n-1}\left(\sum_{j=1}^{i-1} W_{i} \bar{W}_{j+1}+\sum_{j=i}^{n-1} \bar{W}_{i+1} W_{j}\right) .
\end{aligned}
$$

Since

$$
\sum_{i=1}^{n-1} W_{i}+\sum_{i=1}^{n-1} \bar{W}_{i+1}=\sum_{i=1}^{n-1} W_{n}=(n-1) W_{n}
$$

we obtain the first inequality in (10). Since $r, s$ are nonnegative real numbers and obviously

$$
2 n+\frac{1}{W_{n}^{2}} \sum_{i=1}^{n-1}\left(\sum_{j=1}^{i-1} W_{i} \bar{W}_{j+1}+\sum_{j=i}^{n-1} \bar{W}_{i+1} W_{j}\right) \geq 0
$$

the second inequality in (10) immediately follows.

Using identity (6) and the triangle inequality, we can establish bounds for the Čebyšev's functional (or Čebyšev's difference) of the Mercer type in terms of the discrete Ostrowski inequality.

Throughout the rest of the paper, let $[a, b]$ and $[c, d]$ be intervals in $\mathbb{R}$, where $a<b, c<d$.

Theorem 2 Let $n \geq 2$ and let $\boldsymbol{w}$ be a real $n$-tuple such that conditions (1) are fulfilled. Then for any real $n$-tuples $\boldsymbol{x} \in[a, b]^{n}, \boldsymbol{y} \in[c, d]^{n}$ the following inequalities hold:

$$
\begin{aligned}
\mid a c+b d- & \frac{1}{W_{n}} \sum_{i=1}^{n} w_{i} x_{i} y_{i}-\left(a+b-\frac{1}{W_{n}} \sum_{i=1}^{n} w_{i} x_{i}\right)\left(c+d-\frac{1}{W_{n}} \sum_{i=1}^{n} w_{i} y_{i}\right) \mid \\
\leq & (d-c)\left[\frac{1}{W_{n}}\left(\sum_{i=1}^{n} W_{i}\left|x_{1}-a\right|+\sum_{i=1}^{n} \bar{W}_{i}\left|b-x_{n}\right|\right)+\sum_{i=1}^{n-1}\left|\Delta x_{i}\right|\right. \\
& \left.+\frac{1}{W_{n}^{2}} \sum_{i=1}^{n-1}\left(\sum_{j=1}^{i-1} W_{i} \bar{W}_{j+1}+\sum_{j=i}^{n-1} \bar{W}_{i+1} W_{j}\right)\left|\Delta x_{i}\right|\right] \\
\leq(b-a)(d-c) & {\left[2 n+\frac{1}{W_{n}^{2}} \sum_{i=1}^{n-1}\left(\sum_{j=1}^{i-1} W_{i} \bar{W}_{j+1}+\sum_{j=i}^{n-1} \bar{W}_{i+1} W_{j}\right)\right] . }
\end{aligned}
$$

Proof Using identity (6) and the triangle inequality, we have

$$
\begin{gathered}
\left|a c+b d-\frac{1}{W_{n}} \sum_{i=1}^{n} w_{i} x_{i} y_{i}-\left(a+b-\frac{1}{W_{n}} \sum_{i=1}^{n} w_{i} x_{i}\right)\left(c+d-\frac{1}{W_{n}} \sum_{i=1}^{n} w_{i} y_{i}\right)\right| \\
\leq\left|x_{1}-a\right|\left|d-y_{n}\right|+\left|b-x_{n}\right|\left|y_{1}-c\right| \\
+\frac{1}{W_{n}}\left[\sum_{i=1}^{n-1} W_{i}\left|x_{1}-a\right|\left|\Delta y_{i}\right|+\sum_{i=1}^{n-1} \bar{W}_{i+1}\left|b-x_{n}\right|\left|\Delta y_{i}\right|\right.
\end{gathered}
$$




$$
\begin{aligned}
& \left.+\sum_{i=1}^{n-1} W_{i}\left|\Delta x_{i}\right|\left|y_{1}-c\right|+\sum_{i=1}^{n-1} \bar{W}_{i+1}\left|\Delta x_{i}\right|\left|d-y_{n}\right|\right] \\
& +\frac{1}{W_{n}^{2}} \sum_{i=1}^{n-1}\left(\sum_{j=1}^{i-1} W_{i} \bar{W}_{j+1}\left|\Delta x_{i}\right|\left|\Delta y_{j}\right|+\sum_{j=i}^{n-1} \bar{W}_{i+1} W_{j}\left|\Delta x_{i}\right|\left|\Delta y_{j}\right|\right)
\end{aligned}
$$

because $W_{i}$ and $\bar{W}_{i}$ are nonnegative for all $i=1, \ldots, n$. Since $\left|y_{1}-c\right|,\left|d-y_{n}\right|,\left|\Delta y_{i}\right|$ for all $i=1, \ldots, n$, are less or equal to $d-c$, and $\left|x_{1}-a\right|,\left|b-x_{n}\right|,\left|\Delta x_{i}\right|$ for all $i=1, \ldots, n$, are less or equal to $b-a$, we obtain inequalities (11).

Remark 1 If in Theorem 2 we add assumption that $R, S$ are nonnegative real numbers such that

$$
\begin{aligned}
& \left|x_{1}-a\right| \leq R, \quad\left|b-x_{n}\right| \leq R, \quad\left|\Delta x_{i}\right| \leq R, \quad i=1, \ldots, n-1, \\
& \left|y_{1}-c\right| \leq S, \quad\left|d-y_{n}\right| \leq S, \quad\left|\Delta y_{i}\right| \leq S, \quad i=1, \ldots, n-1,
\end{aligned}
$$

then we obtain refinements of the two inequalities proved in [5] under the same assumption. Namely, we have inequalities

$$
\begin{aligned}
\left|a c+b d-\frac{1}{W_{n}} \sum_{i=1}^{n} w_{i} x_{i} y_{i}-\left(a+b-\frac{1}{W_{n}} \sum_{i=1}^{n} w_{i} x_{i}\right)\left(c+d-\frac{1}{W_{n}} \sum_{i=1}^{n} w_{i} y_{i}\right)\right| \\
\leq S\left[\frac{1}{W_{n}}\left(\sum_{i=1}^{n} W_{i}\left|x_{1}-a\right|+\sum_{i=1}^{n} \bar{W}_{i}\left|b-x_{n}\right|\right)+\sum_{i=1}^{n-1}\left|\Delta x_{i}\right|\right. \\
\left.+\frac{1}{W_{n}^{2}} \sum_{i=1}^{n-1}\left(\sum_{j=1}^{i-1} W_{i} \bar{W}_{j+1}+\sum_{j=i}^{n-1} \bar{W}_{i+1} W_{j}\right)\left|\Delta x_{i}\right|\right] \\
\leq R S\left[2 n+\frac{1}{W_{n}^{2}} \sum_{i=1}^{n-1}\left(\sum_{j=1}^{i-1} W_{i} \bar{W}_{j+1}+\sum_{j=i}^{n-1} \bar{W}_{i+1} W_{j}\right)\right]
\end{aligned}
$$

and, as a special case when $w_{i}=1(i=1, \ldots, n)$, we have inequalities

$$
\begin{aligned}
& \left|a c+b d-\frac{1}{n} \sum_{i=1}^{n} x_{i} y_{i}-\left(a+b-\frac{1}{n} \sum_{i=1}^{n} x_{i}\right)\left(c+d-\frac{1}{n} \sum_{i=1}^{n} y_{i}\right)\right| \\
& \quad \leq S\left(\frac{n+1}{2}\left(\left|x_{1}-a\right|+\sum_{i=1}^{n-1}\left|\Delta x_{i}\right|+\left|b-x_{n}\right|\right)-\frac{1}{2 n} \sum_{i=1}^{n-1} i(n-i)\left|\Delta x_{i}\right|\right) \\
& \leq R S \frac{(n+1)(5 n+7)}{12} .
\end{aligned}
$$

\section{Bounds for the Jensen-Mercer functional}

Jensen-Mercer inequality

$$
f\left(a+b-\frac{1}{W_{n}} \sum_{i=1}^{n} w_{i} x_{i}\right) \leq f(a)+f(b)-\frac{1}{W_{n}} \sum_{i=1}^{n} w_{i} f\left(x_{i}\right),
$$


for a convex function $f:(\alpha, \beta) \rightarrow \mathbb{R}$, real $n$-tuple $\boldsymbol{x} \in[a, b]^{n}$, and positive real $n$-tuple $\boldsymbol{w}$, where $-\infty \leq \alpha<a<b<\beta \leq \infty$, was proved in [6]. In [1], it was proved that it remains valid when $\boldsymbol{x}$ is monotonic and $\boldsymbol{w}$ satisfies conditions (1).

Using our results from the previous section, we establish Ostrowski-like bounds for the Jensen-Mercer functional, i.e., the difference of the right- and left-hand sides of inequality (15).

Theorem 3 Let $f:(\alpha, \beta) \rightarrow \mathbb{R}$ be a differentiable function and suppose that $\gamma, \delta$ are real numbers such that $\gamma \leq f^{\prime}(x) \leq \delta$, for all $x \in(\alpha, \beta)$. Let $n \geq 2$ and suppose that $n$-tuple $\boldsymbol{x} \in[a, b]^{n}$, where $-\infty \leq \alpha<a<b<\beta \leq \infty$, satisfies conditions (12). Let $\boldsymbol{w}$ be a real $n$ tuple such that conditions (1) are fulfilled and $a+b-\frac{1}{W_{n}} \sum_{i=1}^{n} w_{i} x_{i} \in[a, b]$. Then

$$
\begin{aligned}
\mid f(a) & +f(b)-\frac{1}{W_{n}} \sum_{i=1}^{n} w_{i} f\left(x_{i}\right)-f\left(a+b-\frac{1}{W_{n}} \sum_{i=1}^{n} w_{i} x_{i}\right) \mid \\
\leq & (\delta-\gamma)\left[\frac{1}{W_{n}}\left(\sum_{i=1}^{n} W_{i}\left|x_{1}-a\right|+\sum_{i=1}^{n} \bar{W}_{i}\left|b-x_{n}\right|\right)+\sum_{i=1}^{n-1}\left|\Delta x_{i}\right|\right. \\
& \left.+\frac{1}{W_{n}^{2}} \sum_{i=1}^{n-1}\left(\sum_{j=1}^{i-1} W_{i} \bar{W}_{j+1}+\sum_{j=i}^{n-1} \bar{W}_{i+1} W_{j}\right)\left|\Delta x_{i}\right|\right] \\
\leq & R(\delta-\gamma)\left[2 n+\frac{1}{W_{n}^{2}} \sum_{i=1}^{n-1}\left(\sum_{j=1}^{i-1} W_{i} \bar{W}_{j+1}+\sum_{j=i}^{n-1} \bar{W}_{i+1} W_{j}\right)\right] .
\end{aligned}
$$

Proof By the mean-value theorem, for any $\zeta, \eta \in(\alpha, \beta)$, there exists some $\xi$ between them such that $f(\zeta)-f(\eta)=f^{\prime}(\xi)(\zeta-\eta)$. Hence, choosing $\zeta=x_{i}$ and $\eta=a+b-\frac{1}{W_{n}} \sum_{i=1}^{n} w_{i} x_{i}$, we obtain

$$
f\left(x_{i}\right)-f\left(a+b-\frac{1}{W_{n}} \sum_{i=1}^{n} w_{i} x_{i}\right)=f^{\prime}\left(\xi_{i}\right)\left(x_{i}-(a+b)+\frac{1}{W_{n}} \sum_{i=1}^{n} w_{i} x_{i}\right) .
$$

Multiplying (17) by $-\frac{w_{i}}{W_{n}}$, and then summing over $i$, we have

$$
\begin{aligned}
- & \frac{1}{W_{n}} \sum_{i=1}^{n} w_{i} f\left(x_{i}\right)+f\left(a+b-\frac{1}{W_{n}} \sum_{i=1}^{n} w_{i} x_{i}\right) \\
= & -\frac{1}{W_{n}} \sum_{i=1}^{n} w_{i} x_{i} f^{\prime}\left(\xi_{i}\right)+\frac{1}{W_{n}}(a+b) \sum_{i=1}^{n} w_{i} f^{\prime}\left(\xi_{i}\right)-\frac{1}{W_{n}^{2}} \sum_{i=1}^{n} w_{i} x_{i} \sum_{i=1}^{n} w_{i} f^{\prime}\left(\xi_{i}\right) .
\end{aligned}
$$

Choosing $\zeta=a, \zeta=b$, respectively, and $\eta=a+b-\frac{1}{W_{n}} \sum_{i=1}^{n} w_{i} x_{i}$, we have

$$
\begin{aligned}
& f(a)-f\left(a+b-\frac{1}{W_{n}} \sum_{i=1}^{n} w_{i} x_{i}\right)=f^{\prime}\left(\xi_{a}\right)\left(\frac{1}{W_{n}} \sum_{i=1}^{n} w_{i} x_{i}-b\right), \\
& f(b)-f\left(a+b-\frac{1}{W_{n}} \sum_{i=1}^{n} w_{i} x_{i}\right)=f^{\prime}\left(\xi_{b}\right)\left(\frac{1}{W_{n}} \sum_{i=1}^{n} w_{i} x_{i}-a\right) .
\end{aligned}
$$


Summing the above three equalities, we obtain

$$
\begin{aligned}
f(a)+ & f(b)-\frac{1}{W_{n}} \sum_{i=1}^{n} w_{i} f\left(x_{i}\right)-f\left(a+b-\frac{1}{W_{n}} \sum_{i=1}^{n} w_{i} x_{i}\right) \\
= & -\frac{1}{W_{n}} \sum_{i=1}^{n} w_{i} x_{i} f^{\prime}\left(\xi_{i}\right)+(a+b) \frac{1}{W_{n}} \sum_{i=1}^{n} w_{i} f^{\prime}\left(\xi_{i}\right)-\frac{1}{W_{n}^{2}} \sum_{i=1}^{n} w_{i} x_{i} \sum_{i=1}^{n} w_{i} f^{\prime}\left(\xi_{i}\right) \\
& +f^{\prime}\left(\xi_{a}\right)\left(\frac{1}{W_{n}} \sum_{i=1}^{n} w_{i} x_{i}-b\right)+f^{\prime}\left(\xi_{b}\right)\left(\frac{1}{W_{n}} \sum_{i=1}^{n} w_{i} x_{i}-a\right) \\
= & a f^{\prime}\left(\xi_{a}\right)+b f^{\prime}\left(\xi_{b}\right)-\frac{1}{W_{n}} \sum_{i=1}^{n} w_{i} x_{i} f^{\prime}\left(\xi_{i}\right) \\
& -\left(a+b-\frac{1}{W_{n}} \sum_{i=1}^{n} w_{i} x_{i}\right)\left(f^{\prime}\left(\xi_{a}\right)+f^{\prime}\left(\xi_{b}\right)-\frac{1}{W_{n}} \sum_{i=1}^{n} w_{i} f^{\prime}\left(\xi_{i}\right)\right)
\end{aligned}
$$

Since $\gamma \leq f^{\prime}(x) \leq \delta$, for all $x \in(\alpha, \beta)$, it holds

$$
\begin{aligned}
& \left|f^{\prime}\left(\xi_{1}\right)-f^{\prime}\left(\xi_{a}\right)\right| \leq \delta-\gamma, \quad\left|f^{\prime}\left(\xi_{b}\right)-f^{\prime}\left(\xi_{n}\right)\right| \leq \delta-\gamma \\
& \left|\Delta f^{\prime}\left(\xi_{i}\right)\right| \leq \delta-\gamma, \quad i=1, \ldots, n-1,
\end{aligned}
$$

and inequalities (16) immediately follow from Theorem 2 and Remark 1.

Remark2 An integral variant of identity (5) can be found in [9] and there is a way to obtain integral variants in terms of Riemann-Stieltjes integral of the Jensen-Mercer inequality from the Jensen-Steffensen inequality (see, for example, [2-4]). Hence, our discrete results can be extended to the continuous case.

\section{Acknowledgements}

We would like to thank the reviewers for their effort to read the paper thoroughly and give us very useful suggestions how to improve it.

\section{Funding}

This publication was supported by the University of Split, Faculty of Science and by the Ministry of Education and Science of the Russian Federation (the Agreement number No. 02.a03.21.0008).

Availability of data and materials

Not applicable.

\section{Competing interests}

The authors declare that they have no competing interests.

Authors' contributions

Both authors jointly worked on the results and they read and approved the final manuscript.

\section{Author details}

${ }^{1}$ Faculty of Electrical Engineering, Mechanical Engineering and Naval Architecture, University of Split, Rudjera Boškovića 32, 21000 Split, Croatia. ${ }^{2}$ RUDN University, Miklukho-Maklaya str. 6, 117198 Moscow, Russia.

\section{Publisher's Note}

Springer Nature remains neutral with regard to jurisdictional claims in published maps and institutional affiliations.

Received: 16 June 2020 Accepted: 4 November 2020 Published online: 12 November 2020 
References

1. Abramovich, S., Klaričić Bakula, M., Matić, M., Pečarić, J.: A variant of Jensen-Steffensen's inequality and quasi-arithmetic means. J. Math. Anal. Appl. 307(1), 370-386 (2005)

2. Barić, J., Matković, A.: Bounds for the normalized Jensen-Mercer functional. J. Math. Inequal. 3(4), 529-541 (2009)

3. Ivelić, S., Klaričić Bakula, M., Pečarić, J.: Converse Jensen-Steffensen inequality. Aequ. Math. 82(3), 233-246 (2011)

4. Klaričić Bakula, M., Matić, M., Pečarić, J.: On some general inequalities related to Jensen's inequality. In: Inequalities and Applications. Internat. Ser. Numer. Math., vol. 157, pp. 233-243. Birkhäuser, Basel (2009)

5. Klaričić Bakula, M., Matković, A., Pečarić, J.: Variants of Čebyšev's inequality with applications. J. Inequal. Appl. 2006, Article ID 39692 (2006)

6. Mercer, A.MCD.: A variant of Jensen's inequality. JIPAM. J. Inequal. Pure Appl. Math. 4(4), Article 73 (2003)

7. Pečarić, J., Tepeš, B.: Improvement of a Grüss type inequality of vectors in normed linear spaces and applications. Rad Hrvat. Akad. Znan. Umjet. Mat. Znan. 15(491), 129-137 (2005)

8. Pečarić, J.E.: On the Čebyšev inequality. Bul. Ştiinţ. Tehn. Inst. Politehn. "Traian Vuia” Timişoara Ser. Mat. Fiz. 25(39)(1980)(1), 5-9 (1981)

9. Pečarić, J.E.: On the Ostrowski generalization of Čebyšev's inequality. J. Math. Anal. Appl. 102(2), $479-487$ (1984)

\section{Submit your manuscript to a SpringerOpen ${ }^{\odot}$ journal and benefit from:}

- Convenient online submission

Rigorous peer review

- Open access: articles freely available online

High visibility within the field

- Retaining the copyright to your article

Submit your next manuscript at $\gg$ springeropen.com 